\title{
57. On Some Types of Polyhedra
}

\author{
By Keiô Nagami \\ Department of Mathematics, Ehime University \\ (Comm. by K. KunUGI, M.J.A., April 12, 1956)
}

Let $Q$ be a class of spaces having some topological property. According to $O$. Hanner [1], a space $Y$ is called respectively an extensor for Q-spaces (an $E S(Q)$ ) or a neighborhood-extensor for Qspaces (an $N E S(Q)$ ) if every $Y$-valued mapping (=continuous transformation) defined on any closed subset $C$ of any $Q$-space $X$ always allows a continuous extension to the whole space $X$ or to an open set $G$ which contains $C$; and a space $Y$ is called respectively an absolute retract for $Q$-spaces (an $A R(Q)$ ) or an absolute neighborhood retract for $Q$-spaces (an $A N R(Q)$ ) if $Y$ is a $Q$-space and is a retract or a neighborhood retract of any $Q$-space containing $Y$ as a closed subset. Analogously to these definitions we generalize the definition of an absolute $n$-retract which was given by C. Kuratowski [2] as follows: A space $Y$ is called an $n-E S(Q)$ if every $Y$-valued mapping defined on any closed subset $C$ of any $Q$-space $X$ with an arbitrary small open set $G \supset C$ such that $\operatorname{dim}(X-G) \leqq n$, always allows a continuous extension to the whole space $X$; and a space $Y$ is called an $n-A R(Q)$ if $Y$ is a $Q$-space and is a retract of any $Q$-space or $X$ containing $Y$ as a closed subset where $\operatorname{dim}(X-G) \leqq n$ holds for an arbitrary small open set $G \supset C$. When $Q$ is a class of metric spaces or of normal spaces, a $Q$-space $Y$ which is an $n-E S(Q)$ is an $n$ $A R(Q)$ and conversely: This is essentially proved in [1, Theorem 8.1]. An $n$-sphere is a well-known example which is an $n$ - $A R$ (normal) [3, Theorem 6.1]. We shall study some types of polyhedra which are $n-E S(Q)$ for the case when $Q$ is a class of metric spaces or of normal spaces.

Let $P=\left\{p_{\alpha}\right\}$ be an abstract set of points with $|P| \geqq n+1$, which will be called a vertex-set, where $n$ is an arbitrary positive integer. The complex with the weak topology spanned by all $m$-simplexes, $m \leqq n$, whose vertices are mutually different points of $P$ is called an $n$-full-polyhedron based on $P$ and is denoted by $K(n, P)$. An $n$ sphere or an $n$-simplex is respectively nothing but an $n$-full-polyhedron based on $P$ with $|P|=n+2$ or with $|P|=n+1$.

Theorem 1. An n-full-polyhedron $K(n, P)$ is an n-ES (metric) for an arbitrary infinite vertex-set $P=\left\{p_{\alpha} ; \alpha \in A\right\}$.

Proof. Let $X$ be a metric space, $C$ be a closed subset of $X$ with an arbitrary small open set $D$ with $\operatorname{dim}(X-D) \leqq n$ and $f$ be 
a mapping of $C$ into $K(n, P)$. Let $f_{a}$ be a non-negative real-valued function of $C$ such that $f_{\alpha}(x)$ is a barycentric weight of $f(x)$ on $p_{\alpha}$. Then $f_{\alpha}$ is continuous and $\left\{U_{\alpha}=\left\{x ; f_{\alpha}(x)>0\right\} ; \alpha \in A\right\}$ is an open covering of $C$ whose order is at most $n+1$. First we shall show that there exists a locally finite open covering $\left\{V_{\alpha} ; \alpha \in A\right\}$ of $X$ whose order is at most $n+1$ such that $V_{\alpha} \frown C=U_{\alpha}$ for every $\alpha \in A$. Since any simplicial polyhedron with the weak topology is an NES (metric) [1, Theorem 25.1], $f$ is continuously extended to $g$ defined on some open set $G$ with $G \supset C$. Let $g_{\alpha}$ be a non-negative real-valued function of $G$ such that $g_{\alpha}(x)$ is a barycentric weight of $g(x)$ on $p_{\alpha}$. Then $\left\{W_{\alpha}=\left\{x ; g_{\alpha}(x)>0\right\} ; \alpha \in A\right\}$ is an open covering of $G$ whose order is at most $n+1$. Let $D \supset C$ be an open set of $X$ with $\operatorname{dim}(X-D) \leqq n$. Let $H$ and $F$ be closed in $X$ and $E$ be open in $X$ with $D \subset F \subset E$ $\subset H \subset G$. Then an open covering $\left\{\left(W_{\alpha}-D\right) \smile(X-H) ; \alpha \in A\right\}$ of $X-D$ can be refined by a locally finite open covering $\left\{B_{\alpha} ; \alpha \in A\right\}$ of $X-D$ whose order is at most $n+1$ such that $B_{a} \subset\left(W_{a}-D\right) \cup(X-H)$ for every $\alpha \in A$. Since $\left\{W_{\alpha}\right\}$ is locally finite in $G$ [1, Lemma 25.4], $\left\{V_{\alpha}=\left(W_{\alpha} \frown E\right) \smile\left(B_{\alpha}-F\right) ; \alpha \in A\right\}$ is, as can easily be seen, a desired one.

Let $h_{\alpha}$ be a non-negative real-valued continuous function of $X$ such that i) $h_{\alpha} \mid C=f_{\alpha}$, ii) $h_{\alpha}(x)=0$ if $x \in X-\left(C \cup V_{\alpha}\right)$, iii) $\left\{x ; h_{\alpha}(x)>0\right\}$ $=V_{\alpha}$. Let $h: X \rightarrow K(n, P)$ be a transformation such that $h(x)$ is the center of gravity of the vertices of $\left\{p_{\alpha} ; x \in V_{\alpha}\right\}$ with the weights $h_{\alpha}(x) / \sum_{\beta \in A} h_{\beta}(x)$. Then $h$ is continuous and satisfies $h \mid C=f$, which completes the proof.

Using the fact that every finite simplicial polyhedron is an NES (normal) [1, Theorem 27.4], we get the following theorem by the quite analogous method used in the above.

Theorem 2. Every finite n-full-polyhedron is an n-ES (normal).

Theorem 3. A finite simplicial polyhedron $L$ with $\operatorname{dim} L \leqq n$ is an $n$-ES (normal) if and only if $L$ is a retract of a finite $n$-fullpolyhedron.

This is almost evident from the fact that $L$ can be imbedded homeomorphically, as a subcomplex, into a finite $n$-full-polyhedron based on $P$ which consists of suitable many vertices. On the other hand, the following analogous theorem is not trivial since a simplicial polyhedron with the weak topology is not always metrizable.

Theorem 4. An infinite simplicial polyhedron $L$ with the weak topology such that $\operatorname{dim} L \leqq n$ is an $n$-ES (metric) if and only if $L$ is a retract of $K(n, P(L))$, where $P(L)$ denotes a set of all vertices of $L$.

Proof. Since if-part is evident, we shall prove only-if-part. Suppose that $L$ is an $n$-ES (metric) and consider $L$ as a subcomplex 
of $K=K(n, P(L))$. Let $\left\{s_{\lambda} ; \lambda \in \Lambda\right\}$ be a collection of all (closed) $n$ simplexes which are not contained in $L$ and suppose that $\Lambda$ is a well-ordered set which consists of all ordinals less than some fixed ordinal $\eta$. Since $L$ is an $n-E S$ (metric), there exists a retract-mapping $f_{1}: L_{1}=L \smile s_{1} \rightarrow L$. Let $L_{\lambda}=L \smile\left\{s_{\xi} ; \xi \leqq \lambda\right\}, \mu$ be some fixed ordinal with $1<\mu<\eta$ and put the transfinite induction assumption that there exist retract-mappings $f_{\nu}: L_{\nu} \rightarrow L, \nu<\mu$, such that $f_{\nu} \mid L_{\xi}=f_{\xi}$ for any $\nu$ and $\xi$ with $\xi<\nu$. Let us show the existence of a retract-mapping $f_{\mu}: L_{\mu} \rightarrow L$ such that $f_{\mu} \mid L_{\nu}=f_{\nu}$ for any $\nu<\mu$. Since $F=s_{\mu} \frown\left(L \cup\left\{s_{\nu}\right.\right.$; $\nu<\mu\})$ is a finite subpolyhedron of $s_{\mu}$, we can choose a $\nu_{0}<\mu$ with $F=s_{\mu} \frown L_{v_{0}}$. Then $f_{v_{0}} \mid F$ has a continuous extension $g: s_{\mu} \rightarrow L$. Let $f_{\mu}: L_{\mu} \rightarrow L$ be a transformation such that i) $f_{\mu} \mid s_{\mu}=g$, ii) $f_{\mu}\left|s_{\nu}=f_{\nu}\right| s_{\nu}$ when $\nu<\mu$, iii) $f_{\mu} \mid L=$ the identity mapping. Then $f_{\mu}$ is continuous since the topology of $K$ is the weak one. Moreover, it is evident that $f_{\mu} \mid L_{\nu}=f_{\nu}$ for every $\nu<\mu$, which completes the transfinite induction. Now we can construct for any $\lambda<\eta$ a retract-mapping $f_{\lambda}: L_{\lambda} \rightarrow L$ such that $f_{\mu} \mid L_{\nu}=f_{\nu}$ for any $\mu$ and $\nu$ with $\nu<\mu<\eta$. Let $f: K \rightarrow L$ be a transformation such that i) $f\left|s_{\lambda}=f_{\lambda}\right| s_{\lambda}$ for any $\lambda<\eta$, ii) $f \mid L=$ the identity mapping, and then $f$ is continuous. Therefore $L$ is a retract of $K$, which completes the proof.

\section{References}

[1] O. Hanner: Retraction and extension of mappings of metric and non-metric spaces, Ark. Mat., 2, 315-360 (1952).

[2] C. Kuratowski: Sur les espaces localement connexes et péaniens en dimension n, Fund. Math., 24, 269-287 (1935).

[3] K. Morita: On the dimension of normal spaces II, J. Math. Soc. Japan, 2, 16$33(1950)$. 